\title{
EUS-Guided Antitumor Therapy for Pancreatic Tumors
}

\author{
Dong Wan Seo \\ Department of Gastroenterology, Asan Medical Center, University of Ulsan College of Medicine, Seoul, Korea
}

Endoscopic ultrasound (EUS) is a very useful modality for the diagnosis and staging of pancreatic masses. With the advent of EUS-guided fine-needle aspiration technology, this modality has made a tremendous leap from imaging modality to histologic diagnosis and therapeutic intervention. EUS offers high-resolution images of and unparalleled access to the pancreas. After locating the tip of the echoendoscope in the duodenum or stomach, several drugs or local treatment modalities can be delivered directly into the pancreas. EUS-guided ethanol lavage with/without paclitaxel injection has been tested for the treatment of cystic tumors of the pancreas, with complete resolution of cystic tumor being observed in up to $70-80 \%$ of patients. Ethanol injection is also performed for the management of solid neuroendocrine tumors of the pancreas. Various type of EUS-guided injection have also been investigated for the treatment of pancreatic cancer. An activated allogenic mixed lymphocyte culture (Cytoimplant) was injected in patients with advanced pancreatic cancer. A replication-deficient adenovirus vector carrying the tumor necrosis factor-alpha gene was also delivered intratumorally by EUS. ONYX-015 is an oncolytic attenuated adenovirus that exhibits replication preferentially in malignant cells, causing cell death, and this has also been injected into pancreatic cancers under EUS guidance. EUS-guided local ablation therapies such as radiofrequency ablation, photodynamic therapy, and brachytherapy are also under investigation. EUS-guided fine-needle injection for various solid or cystic lesions is a rapidly expanding field. This article reviews the various applications of EUS for the treatment of pancreatic tumors. (Gut Liver 2010;4(Suppl. 1):S76-81)

Key Words: Endoscopic ultrasonography; Pancreas cancer; Cystic tumor; Radiofrequency ablation; Photody- namic therapy; Brachytherapy; Cytoimplant; Immunotherapy

\section{INTRODUCTION}

Endoscopic ultrasonography (EUS) was introduced to clinical medicine for more than 30 years $\mathrm{ago}^{1-3}$ and has contributed to the diagnosis and management of pancreatic tumors. EUS scanning system can be divided into two categories: radial and linear, with both mechanical and electronic formats available in each. With the advent of linear-array echoendoscope, it is possible to trace needle tract under ultrasound and to do EUS-guided fine needle aspiration (EUS-FNA). Recently, EUS-guided interventions are tried using various modifications of this technology. Interventional applications of EUS are also tried for pancreatic diseases such as pseudocyst drainage, pancreatic pain control, cystic tumor ablation and solid tumor therapy. In this review, we are going to focus particularly on EUS-guided treatment of benign and malignant pancreatic tumors.

\section{EUS-GUIDED ABLATION OF PANCREATIC CY- STIC TUMOR}

Pancreatic cystic tumors encompass a wide spectrum of histopathologies and biologic behaviors. Although large sized and symptomatic cystic tumors were common in the past, incidentally detected small cystic tumors are more common these days. In an autopsy study, the prevalence of pancreatic cyst was reported up to $25 \%$, with increasing prevalence paralleling advanced patient age. ${ }^{4}$ Commonly encountered histopathogies are mucinous cystic neoplasm (MCN), serous cystic neoplasm (SCN), and intra-

\footnotetext{
Correspondence to: Dong Wan Seo

Department of Gastroenterology, Asan Medical Center, 388-1 Pungnap 2-dong, Songpa-gu, Seoul 138-736, Korea

Tel: +82-2-3010-3190, Fax: +82-2-485-5782, E-mail: dwseoamc@amc.seoul.kr

DOI: $10.5009 /$ gnl.2010.4.S1.S76
} 
ductal papillary mucinous neoplasm (IPMN). Non-mucinous cystic lesions such as serous cystadenoma mostly show benign histology and biologic behavior. Mucinous cystic tumors can show benign, borderline, or malignant histology and are regarded as premalignant or malignant tumor. For the treatment of mucinous cystic tumors, generally surgical resection is recommended. Management of pancreatic cystic tumor is challenging as most are asymptomatic but may have malignant potential, and surgical resection of cystic neoplasms of the pancreas has a substantial morbidity of $20 \%$ to $40 \%$ and a mortality of $2 \% .^{5-7}$ As an alternative treatment, EUS-guided ethanol lavage was tried. Ethanol is the most commonly used ablative agent for renal and hepatic cysts. ${ }^{8-10}$ Its ablative effects on cyst epithelium are induced by cell-membrane lysis, protein denaturation and vascular occlusion. ${ }^{11}$ Under EUS guidance, cystic tumor was punctured using a fine needle and cystic fluid was aspirated. After complete aspiration of cystic fluid, ethanol was injected into the cystic cavity and injected ethanol was re-aspirated after 3-5 minutes of retention time (Fig. 1). An initial pilot study ${ }^{12}$ showed that EUS-guided ethanol lavage for pancreatic cystic tumors is safe and feasible, with complete resolution being achieved in 8 of 25 patients (33\%) and variable histologic degrees of epithelial ablation observed in all resected specimens. To improve the complete resolution rate, our group combined taxol injection after ethanol lavage. ${ }^{13,14}$ Paclitaxel, a widely used chemotherapeutic agent, is so highly hydrophobic and viscous in nature that it can exert its effect for a longer period of time within a closed cystic cavity with low possibility of leakage through the puncture site. A preliminary study ${ }^{13}$ found that 11 of 14 patients showed complete cyst resolution after ethanol lavage and paclitaxel injection. Primary dis-
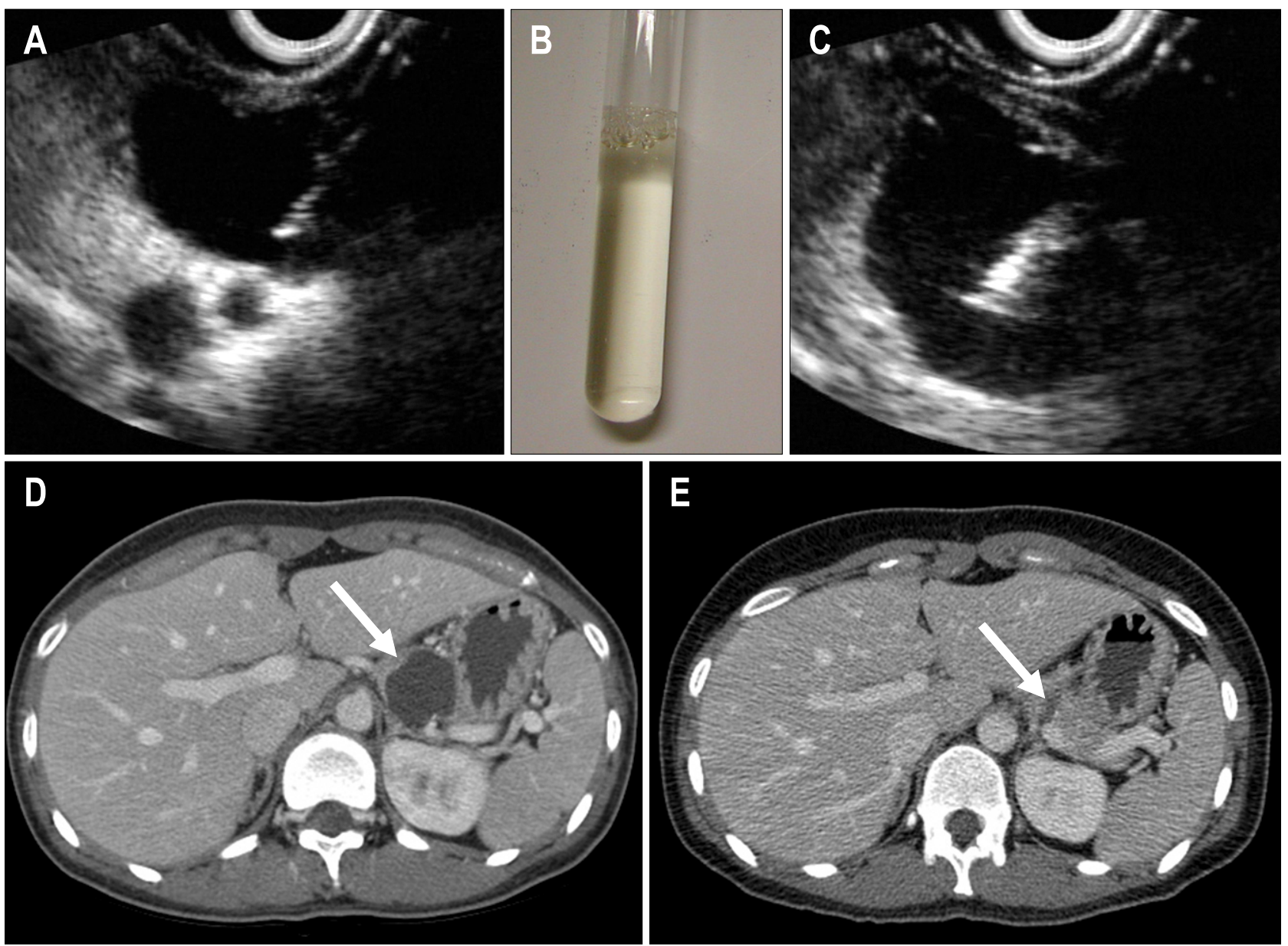

Fig. 1. Endoscopic ultrasonography (EUS)-guided pancreatic cystic tumor ablation. (A) For the ablation of pancreatic cystic tumor, a fine, 22-gauge needle was inserted into the cystic cavity. (B) The cystic fluid was aspirated and found to be clear. (C) After cystic fluid aspiration, 99\% ethanol was injected into the cavity and left for 5 minutes to cause epithelial ablation. (D) CT scan before EUS-guided treatment. A cystic tumor $(3.5 \times 3.1 \times 2.5 \mathrm{~cm}$ ) was located in the body of the pancreas (arrow). (E) CT scan taken 6 months after EUS-guided treatment, showing that the cystic tumor had disappeared (arrow). 
tortion of cyst epithelial lining by ethanol lavage and secondary inhibition by easily diffused paclitaxel may result in a synergistic ablative effect from the combination of these two agents. Previous studies have involved only a small number of patients and short-term outcomes. ${ }^{12-14}$ Therefore, further studies involving a large study population with long-term follow up are required.

\section{EUS-GUIDED ETHANOL ABLATION OF INSULI- NOMA}

Pancreatic insulinomas are usually small in size and present with recurrent hypoglycemic symptoms. EUS gives high resolution image of the pancreas and can detect small hypoechoic mass in the pancreas. Although pancreatic surgery is considered a first-line approach for insulinoma treatment, a subset of patients are not suitable for pancreatic surgery because of old age or comorbid illness. Ethanol injection treatment was tried successfully for patients with hepatocelluar carcinoma or hepatic metastasis. Scott et al. ${ }^{15}$ described the successful percutaneous ablation of hepatic metastasis of insulinoma. Jurgensen et al. ${ }^{16}$ reported successful EUS-guided ablation of pancreatic neuroendocrine tumor by ethanol injection. They achieved complete resolution based on clinical, morphologic and biochemical data. This method is also tried for left adrenal metastasis of non-small cell lung cancer to cause ablation of metastatic mass and to provide palliation of cancer-related pain. ${ }^{17}$ Based on previously reported case series, EUS guided ablation by ethanol injection seems to be a possible alternative treatment for benign pancreatic mass. However, there are several unsolved issues; optimal type and size of injection needle, safe target area within tumor mass, and the adequate amount of alcohol to achieve successful ablation without causing significant pancreatitis. Further studies involving larger number of patients are required.

\section{EUS-GUIDED CELIAC PLEXUS NEUROLYSIS}

Celiac ganglion receives afferent fibers from pancreas and upper abdominal viscera and conveys pain sense via splanchnic nerve to central nervous system. To control pancreatic pain or retroperitoneal pain, celiac plexus neurolysis has been performed under fluoroscopic guidance or CT guidance. It can be either bilateral an anterior or a posterior approach. However, these approaches should traverse a long needle tract and can cause serious complications such as paraplegia. EUS-guided celiac plexus neurolysis has been introduced as an alternative approach and is now widely used for palliation of pain in patients with pancreatic cancer. Under linear-array echoendoscope, abdominal aorta and celiac trunk can be traced from lesser curvature of the stomach. Celiac ganglia are located around celiac trunk and subtle clockwise or counterclockwise rotation can delineate small hypoechoic ganglia in some cases. Bupivacaine is injected at first to control pain related to ethanol injection. Then ethanol can be injected directly into the ganglia or around celiac trunk. Earlier report ${ }^{18}$ showed $78 \%$ improvement in pain score at a median follow-up of 10 weeks. Levy et al. ${ }^{19}$ retrospectively reviewed 33 patients with pancreatic cancer or chronic pancreatitis who received EUS guided celiac plexus neurolysis or celiac plexus block directly into the celiac ganglia. Twelve patients experienced pain during or immediately after the procedure. Effective pain relief was more frequently observed in patients who developed pain compared with those who did not. Complications of EUS guided celiac plexus neurolysis are minor such as transient diarrhea and hypotension. Based on the present data, EUS guided celiac plexus neurolysis for pancreatic cancer pain can be offered safely with high immediate success rate. However, the limitation of this approach is duration of effectiveness and pain increased again in most cases 2 or 3 months after treatment.

\section{EUS-GUIDED DEUVERY OF ANTI-TUMOR AGENTS}

Pancreatic ductal adenocarcinoma is one of the most difficult tumor to treat and has an extremely poor prognosis. Most patients die within a year or two after diagnosis and the overall 5 year survival rate is less than $5 \%$ even after extensive treatment including surgery, chemotherapy and/or radiation therapy. Pancreatic cancer is one of the main causes of cancer-related deaths in most countries and the annual incidence rates approach nearly to annual death rates. For the control of this dismal behavior, various materials were tried by several endosonographers. It seems to be very attractive approach to inject of anti-tumor agent under EUS guidance such as chemotherapeutic drugs, anti-tumor virus, or immature dendritic cells. EUS-guided injection of anti-tumor agent was first attempted in the pancreas because EUS-guided approach is a simple, effective and safer than CT-guided approach. EUS can visualize not only the tumor mass but also complex anatomy around pancreas and administration of anti-tumor agent in real time. After localization of pancreatic cancer under EUS, interposing vessel can be identified by applying Doppler signal and EUS can guide the angle of puncture without causing vascular injury. While maintaining slow and steady injection, needle insertion and withdrawal can be repeated within the 
tumor mass at slightly different angle for even distribution of anti-tumor agent.

\section{Allogenic mixed lymphocyte culture (cytoim- plant) in pancreatic cancer}

Chang et al. ${ }^{20}$ tried to inject cytoimplant directly into pancreatic cancer and evaluated the technical feasibility and safety. In phase I trial, 8 patients with unresectable pancreatic cancer received EUS-guided injection of cytoimplant. Cytoimplant cells were delivered by a single EUS-guided injection and doses were escalated over the study period. The first two patients received 3 billion cells, the next three patients received 6 billion cells and the last three patients received 9 billion cells. The median survival was 13.2 months, with 2 partial responders and 1 minor response. There was no serious complication and the most common side effect was low-grade fever without leukocytosis, which was managed effectively by acetaminophen. Although they showed the technical feasibility and procedural safety, there has been no further study using this agent for more than 10 years.

\section{ONYX-015 in pancreatic cancer}

Hecht et al. $^{21}$ tried EUS-guided ONYX-015 injection in patients with pancreatic cancer. ONYX-015 is an E1B$55-\mathrm{kDa}$ gene-deleted adenovirus which shows replication selectivity and preferentially replicates and kills malignant cells. They injected ONYX-015 in 21 patients over an 8 -week period. Two patients developed sepsis and two patients had duodenal perforation. No patient showed tumor regression by ONYX-015 at day 35. After combination with gemcitabine therapy, two patients showed partial response and two showed minor response. Six patients showed stable disease and 11 showed progressive disease.

\section{TNFerade in pancreatic cancer}

TNFerade is also injected in patients with pancreatic cancer as a novel gene transfer method. ${ }^{22,23}$ TNFerade is a replication-deficient adenovector containing human tumor necrosis factor (TNF)- $\alpha$ gene, regulated by a radiation-inducible promoter Egr-1 (early growth response). The advantage of this approach is the potential to maximize local antitumor activity and to minimize systemic toxicity. Phase I trial designed as a 5-week treatment of weekly intratumoral injections of TNFerade $\left(4 \times 10^{9}, 4 \times 10^{10}\right.$, and $4 \times 10^{11}$ particle units in $2 \mathrm{~mL}$ ) by EUS guidance. Up to 4 injections were given by EUS and TNFerade was combined with 5-FU and radiation. A cohort of 50 patients showed mild toxicities and well tolerated. Compared 2 lower dose cohorts, higher dose cohort showed greater locoregional control of treated tumors, longer pro- gression-free survival, improved median survival $(6.6,8.8$, $11.2,10.9$ months in the $4 \times 10^{9}, 4 \times 10^{10}, 4 \times 10^{11}, 4 \times 10^{12}$ particle unit cohorts, respectively). Although this result seemed to be promising, the phase III randomized controlled trial has not been published yet.

\section{EUS-GUIDED IMMUNOTHERAPY}

Immunotherapy against cancer has been investigated using different type of immune cells and dendritic cells are the most potent antigen-presenting cells for induction of primary T-cell dependent immune responses. Immunotherapy has also been tried for pancreatic cancers. The effect of dendritic cell (DC)-based immunotherapy was investigated and tumor growth was significantly inhibited in hamster model. ${ }^{24}$

Hirooka et l. $^{25}$ undertook the first clinical trial involving a combination therapy of gemcitabine with immunotherapy for patients with inoperable locally advanced pancreatic cancer. Basic idea of this study was combination of chemotherapy with dendritic cell vaccination. They hypothesized that apoptotic cells induced by gemcitabine treatment could release tumor antigens slowly over time and this stimulates dendritic cells to process and present tumor antigens. To enhance the antigen presenting capabilities, OK432-pulsed dendritic cells were used and OK432 is widely used maturation stimulus for dendritic cells. Five patients received combined treatment which consist of intravenous gemcitabine treatment and EUSguided intratumoral injection of OK432-pulsed dentritic cells. To induce additional anticancer activity, the patients also received lymphokine-activated killer cells stimulated with anti-CD3 monoclonal antibody. Three of 5 patients showed effective response to this treatment ( 1 partial remission, 2 stable disease more than 6 months). There was no serious complication related to treatment. This combination of chemotherapy and immunotherapy could be considered to cause synergistic effect and more studies are waited.

\section{EUS-GUIDED TUMOR ABLATION}

Local ablative modalities such as radiofrequency ablation (RFA), ${ }^{26}$ photodynamic therapy (PDT), ${ }^{27}$ or brachytherapy $^{28,29}$ are also applied to pancreas in animal studies or human studies. RFA is primarily delivered to liver percutaneously or intraoperatively. EUS-guided RFA in a pig model proved that this modality can also be delivered to the pancreas transgastrically and cause well-demarcated coagulation zone. ${ }^{26}$ EUS-guided RFA may be a potential modality for the management of small pancreatic tumors. 
This modality, however, should be improved and refined to reduce the possible complications such as pancreatitis, gastric burn or perforation. EUS-guided PDT in a pig model showed that it is possible to pass 19-gauge needle into the liver or pancreas and make localized tissue necrosis without causing significant procedure-related complications. $^{27}$

Radioactive seeds of iodine-125 are injected directly into tumors in patients with advanced pancreatic cancer under EUS-guidance. This approach achieved partial response, minimal disease or stable disease and clinical benefit was seen in $30 \%$ of patients, primarily from reduction of pain. $^{29}$

\section{CONCLUSION}

In conclusion, EUS-guided injection offers minimally invasive access for delivery of local therapeutic modalities including gene therapy, RFA, PDT, brachytherapy, and immunotherapy. EUS-guided antitumor therapy is a continuously evolving field and many studies are currently undergoing. Many of these approaches are still investigational but will play a key role in the treatment of benign and malignant pancreatic disease in the future.

\section{REFERENCES}

1. DiMagno EP, Buxton JL, Regan PT, et al. Ultrasonic endoscope. Lancet 1980;1:629-631.

2. Hisanaga K, Hisanaga A, Nagata K, Ichie Y. High speed rotating scanner for transgastric sonography. AJR Am J Roentgenol 1980;135:627-629.

3. Strohm WD, Phillip J, Hagenmuller F, Classen M. Ultrasonic tomography by means of an ultrasonic fiberendoscope. Endoscopy 1980;12:241-244.

4. Kimura W, Nagai H, Kuroda A, Muto T, Esaki Y. Analysis of small cystic lesions of the pancreas. Int J Pancreatol 1995;18:197-206

5. Allen PJ, D'Angelica M, Gonen M, et al. A selective approach to the resection of cystic lesions of the pancreas: results from 539 consecutive patients. Ann Surg 2006;244: 572-582.

6. Goh BK, Tan YM, Cheow PC, et al. Cystic lesions of the pancreas: an appraisal of an aggressive resectional policy adopted at a single institution during 15 years. Am J Surg 2006;192:148-154.

7. Horvath KD, Chabot JA. An aggressive resectional approach to cystic neoplasms of the pancreas. Am J Surg 1999;178:269-274.

8. Bean WJ. Renal cysts: treatment with alcohol. Radiology 1981;138:329-331.

9. Hanna RM, Dahniya MH. Aspiration and sclerotherapy of symptomatic simple renal cysts: value of two injections of a sclerosing agent. AJR Am J Roentgenol 1996;167:781783.
10. Larssen TB, Viste A, Jensen DK, Sondenaa K, Rokke O, Horn A. Single-session alcohol sclerotherapy in benign symptomatic hepatic cysts. Acta Radiol 1997;38:993-997.

11. Gelczer RK, Charboneau JW, Hussain S, Brown DL. Complications of percutaneous ethanol ablation. J Ultrasound Med 1998;17:531-533.

12. Gan SI, Thompson CC, Lauwers GY, Bounds BC, Brugge WR. Ethanol lavage of pancreatic cystic lesions: initial pilot study. Gastrointest Endosc 2005;61:746-752.

13. Oh HC, Seo DW, Lee TY, et al. New treatment for cystic tumors of the pancreas: EUS-guided ethanol lavage with paclitaxel injection. Gastrointest Endosc 2008;67:636-642.

14. Oh HC, Seo DW, Kim SC, et al. Septated cystic tumors of the pancreas: is it possible to treat them by endoscopic ultrasonography-guided intervention? Scand J Gastroenterol 2009;44:242-247.

15. Scott A, Hinwood D, Donnelly R. Radio-frequency ablation for symptom control in a patient with metastatic pancreatic insulinoma. Clin Endocrinol (Oxf) 2002;56:557-559.

16. Jurgensen C, Schuppan D, Neser F, Ernstberger J, Junghans U, Stolzel U. EUS-guided alcohol ablation of an insulinoma. Gastrointest Endosc 2006;63:1059-1062.

17. Artifon EL, Lucon AM, Sakai P, et al. EUS-guided alcohol ablation of left adrenal metastasis from non-small-cell lung carcinoma. Gastrointest Endosc 2007;66:1201-1205.

18. Wiersema MJ, Wiersema LM. Endosonography-guided celiac plexus neurolysis. Gastrointest Endosc 1996;44:656662.

19. Levy MJ, Topazian MD, Wiersema MJ, et al. Initial evaluation of the efficacy and safety of endoscopic ultrasoundguided direct Ganglia neurolysis and block. Am J Gastroenterol 2008;103:98-103.

20. Chang KJ, Nguyen PT, Thompson JA, et al. Phase I clinical trial of allogeneic mixed lymphocyte culture (cytoimplant) delivered by endoscopic ultrasound-guided fine-needle injection in patients with advanced pancreatic carcinoma. Cancer 2000;88:1325-1335.

21. Hecht JR, Bedford R, Abbruzzese JL, et al. A phase I/II trial of intratumoral endoscopic ultrasound injection of ONYX-015 with intravenous gemcitabine in unresectable pancreatic carcinoma. Clin Cancer Res 2003;9:555-561.

22. Chang KJ, Irisawa A. EUS 2008 Working Group document: evaluation of EUS-guided injection therapy for tumors. Gastrointest Endosc 2009;69(2 Suppl):S54-S58.

23. Chang KJ, Lee JG, Holcombe RF, Kuo J, Muthusamy R, $\mathrm{Wu} \mathrm{ML}$. Endoscopic ultrasound delivery of an antitumor agent to treat a case of pancreatic cancer. Nat Clin Pract Gastroenterol Hepatol 2008;5:107-111.

24. Akiyama Y, Maruyama K, Nara N, et al. Antitumor effects induced by dendritic cell-based immunotherapy against established pancreatic cancer in hamsters. Cancer Lett 2002;184:37-47.

25. Hirooka Y, Itoh A, Kawashima H, et al. A combination therapy of gemcitabine with immunotherapy for patients with inoperable locally advanced pancreatic cancer. Pancreas 2009;38:e69-e74.

26. Goldberg SN, Mallery S, Gazelle GS, Brugge WR. EUS-guided radiofrequency ablation in the pancreas: results in a porcine model. Gastrointest Endosc 1999;50:392401.

27. Yusuf TE, Matthes K, Brugge WR. EUS-guided photo- 
dynamic therapy with verteporfin for ablation of normal pancreatic tissue: a pilot study in a porcine model (with video). Gastrointest Endosc 2008;67:957-961.

28. Jin Z, Du Y, Li Z, Jiang Y, Chen J, Liu Y. Endoscopic ultrasonography-guided interstitial implantation of iodine 125 -seeds combined with chemotherapy in the treatment of unresectable pancreatic carcinoma: a prospective pilot study. Endoscopy 2008;40:314-320.

29. Sun S, Xu H, Xin J, Liu J, Guo Q, Li S. Endoscopic ultrasound-guided interstitial brachytherapy of unresectable pancreatic cancer: results of a pilot trial. Endoscopy 2006; 38:399-403. 